\title{
A Framework for Visualizing Heterogeneous Construction Data Using Semantic Web Standards
}

\author{
Mostafa Ali $(i)$ and Yasser Mohamed \\ University of Alberta, Edmonton, AB, Canada \\ Correspondence should be addressed to Mostafa Ali; mostafaali@ualberta.ca
}

Received 25 January 2018; Revised 2 August 2018; Accepted 13 August 2018; Published 16 September 2018

Academic Editor: Flora Faleschini

Copyright (c) 2018 Mostafa Ali and Yasser Mohamed. This is an open access article distributed under the Creative Commons Attribution License, which permits unrestricted use, distribution, and reproduction in any medium, provided the original work is properly cited.

\begin{abstract}
3D Visualization provides a mean for communicating different construction activities to diverse audiences. The scope, level of detail, and time resolution of the 3D visualization process are determined based on the targeted audiences. Developing the 3D visualization requires obtaining and merging heterogeneous data from different sources (such as BIM model and CPM schedule). The data merging process is usually carried out on ad hoc basis for a specific visualization case which limits the reusability of the process. This paper discusses a framework for automatic merging of heterogeneous data to create a visualization. The paper describes developing an ontology which captures concepts related to the visualization process. Then, heterogeneous data sources that are commonly used in construction are fed into the ontology which can be queried to produce different visualization scenarios. The potential of this approach has been demonstrated by providing multiple visualization scenarios that cover different audiences, levels of detail, and time resolutions.
\end{abstract}

\section{Introduction}

A typical visualization paradigm in construction utilizes Building Information Modeling (BIM) data along with project schedule to depict the construction progress. This approach, known as $4 \mathrm{D}$ visualization, focuses on high-level details only, in which each element simply appears in its final position when the corresponding task in the schedule is complete. This type of visualization provides an appealing interface to illustrate the sequence of activities in the schedule and helps align objectives between different stakeholders [1], understand the schedule, and identify potential problems [2]. However, it fails to give more details about site conditions (e.g., congestions due to temporary facilities) and the interaction between personnel and equipment on-site [3].

A more detailed visualization scenario is operational visualization, which focuses on intricate details rather than the high-level progress. This type of visualization depicts details such as material movement, interaction with cranes, etc. [4]. A successful implementation of operational visualization requires more data than the typical $4 \mathrm{D}$ visualization approach. For example, it requires scaffold logs along 3D model and schedule to visualize scaffold operations.

Bearing in mind that the construction industry is characterized by large volumes of data that come from heterogeneous data sources [5], creating operational visualization is a tedious task that requires mapping and merging these data which is usually performed on an ad hoc basis per specific case study.

Heesom and Mahdjoubi [6] have stated that the flow of data is one of the most critical issues in the development of visualization tools. They argued that most visualization applications require manual input from different data sources, which is a potential reason that it is not widely used in the construction industry.

This paper discusses a framework for generating visualizations of different construction activities with different levels of detail. The proposed approach is data-centric that focuses on merging and processing data rather than the visualization application. 
In order to focus on merging and processing data, the authors developed an ontology [7] that conceptualizes information related to the visualization process. This ontology formalizes data flow from different data sources that need to be visualized.

Afterwards, SPARQL queries [8] have been used to retrieve and manipulate the stored data and automatically generate input files for the visualization engine. Separating the data from the visualization engine allows changing the visualization application without having to edit the ontology or the input data. Additionally, it makes it possible to add new data sources to an existing visualization process without breaking compatibility with the visualization application, as will be shown later.

The remainder of the paper is structured as follows: first, we discuss previous work related to visualization in the construction domain, then we outline the research objectives and methodology and finally discuss in detail our proposed framework and demonstrate it with real case scenarios that cover different applications and different ranges of activities commonly used in the construction domain.

1.1. Visualization in Construction. $3 \mathrm{D}$ visualization of construction projects is challenging due to its complexity and unpredictable nature [9], which leads to many customized applications that are applicable only to a certain type of construction project or even to a specific project.

There are many examples of using visualization in construction domain. For example, it has been stated that a typical construction simulation consists of eight federates [10] and an essential federate of these eight federates is a visualization federate, which shows the simulation progress and results to the end user.

Many examples of using visualization in simulation can be found in the literature. These include tunneling [11], training [12, 13], and crane operations [14, 15].

Outside the simulation realm, visualization applications can be found in virtual reality $[16,17]$, safety $[18,19]$, and transportation $[20,21]$.

In the following sections (1.2-1.4), three previous projects that are closely related to the visualization process and developed by the authors will be discussed. These projects were built upon High-level Architecture- (HLA-) distributed simulation $[22,23]$.

In HLA simulation, the simulated problem is broken into smaller components, known as federates, that interact with each other during the simulation. Each of the mentioned project here has a visualizer federate that shows progress made by other federates. Here, we will focus on the visualization federate in each case by discussing the challenges and lessons learned to show the necessity of a generic visualization process that can accept data from different sources: whether from simulation components or standalone applications.

1.2. Pipe Manufacturing Visualizer. The first project simulates the construction of oil refineries and petrochemical plants, which follow modular construction paradigms.
Different modules are fabricated by assembling components from different trades (e.g., pipes, structural steel, and equipment) in an off-site module yard; then they are shipped to project site and installed using heavy-lift cranes. This is a complex process that involves multiple parties and requires careful planning and coordination.

An HLA-distributed simulation has been developed to model this operation $[24,25]$. The simulation focuses on the piping manufacturing process; it simulates constructing piping spools and assembling them into modules in a module yard, transportation to construction site, and final installation. In addition, it tracks the associated schedule and ensures that predecessors have been fulfilled before installation.

This distributed simulation contains five federates:

(1) Simulation Federate. This simulates all fabrication operations and produces related statistics like the production rate.

(2) Resource Allocation Federate. This allocates available cranes to ready-to-install modules. Crane selection depends on availability and ability to handle the module.

(3) Site Construction Federate. This is responsible for preparing the construction site topography based on a topography data file.

(4) Yard Viewer Federate. This is a $3 \mathrm{D}$ visualizer that displays simulation activities in the module yard.

(5) Site Viewer Federate. This is another 3D visualizer that displays simulation activities in the construction site.

The visualization federates enhance results' readability, give more insight on the piping manufacturing process, and provide an easy way to validate the simulation. The visualizer has been used to provide the following:

(i) Visualize the logical sequence of the schedule as it displays the installation sequence according to the provided schedule.

(ii) Display the utilization of the module yard, which is divided into bays where different pipe modules can be assembled in parallel. The visualizer also helps determine whether the bays are over- or underutilized.

(iii) Combine the schedule logic with the spatial data to show the congestion on the construction site.

1.3. Earthmoving Visualizer. The second case tackles the earthmoving process. Due to its repetitive nature, an earthmoving operation is a good candidate for simulation. Many simulation models have been built to study the effect of different factors on earthmoving operations. This includes fleet optimization [26-28]; decision support [29-31], and utilizing real data [32-34].

Most of these simulation models considered an earthmoving operation as one model, which limits scalability and extensibility (i.e., adding new functionality in next developing cycles); in this project, distributed simulation 
was used to overcome these limitations by breaking the earthmoving operations into six federates: controller, loader, mover, breakdown, weather, and visualizer-[35].

The controller federate is responsible for creating a federation, defining a scenario (e.g., fleet composition, road length, and hauling material), and displaying statistical results (e.g., production rate and utilization). The loader and mover deal with equipment movements in the mine and road, respectively, and this separation allows different teams to focus on different conditions; for example, while mover focused on tire wearing due to rolling resistance, the loader considered queuing trucks in the mine pit. The breakdown federate simulates the breakdown effect on the production rate by breaking down trucks and excavators based on distributions drawn from historical data. The weather federate studies the effect of weather conditions (e.g., precipitation, wind speed, and snow depth) on the earthmoving process, and based on the earthmoving location, the weather federate sends weather conditions to all other federates.

At the simulation inception, the visualizer federate loads terrain and roads from a " $3 \mathrm{ds}$ " file format. Afterwards, it loads trucks and excavators when they are registered by other federates; the visualizer is equipped with $3 \mathrm{D}$ assets library for many trucks' and excavators' models, and it loads the required model based on other federates' requests. During the simulation, the trucks' position and state (i.e., loaded vs. empty, working vs. broken down) are interpreted and displayed based on other federates' updates.

The visualizer federate provides insight into the earthmoving operation; it shows the truck movement and state as seen in Figure 1. The user can manipulate (i.e., zooming, panning, and rotating) the model. $\mathrm{He} / \mathrm{she}$ can also hover over each piece of equipment to show its properties.

This project shows how important and necessary a visualizer is to introduce simulation results to a nonexpert in a more intuitive way. Nonetheless, this project shows several drawbacks in its visualization structure. For example, it has been built upon a custom visualization interface rather than a COTS visualization engine, which in turn leads to a relatively slow visualizer with inability to handle sophisticated $3 \mathrm{D}$ assets. It also has been built for one visualization scenario which limits its expansion to other scenarios; this can be noticed in its data model that describes specific classes such as trucks and excavators. Additionally, it shows simulation results, but they cannot be filtered or queried to visualize different visualization scenarios.

1.4. Distributed Observer Network (DON). The Simulation Exploration Experience (SEE) [36] is an annual event organized by The National Aeronautics and Space Administration (NASA) and The Society for Modeling \& Simulation International. SEE invites students from different universities around the world along with industry and professional associates to develop a distributed simulation for a space mission. The SEE challenge, with a time frame of around six months, gives students an inspiring way to learn and apply HLA standards while collaborating with students from other universities around the world.

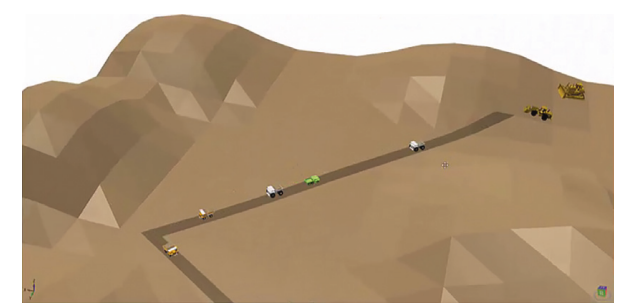

Figure 1: Earthmoving operation as displayed in the visualizer.

The SEE 2015 challenge (SEE 2015) required developing a distributed simulation of a lunar mission. Teams from eight universities (University of Alberta, University of Bordeaux, University of Brunel, University of Calabria, University of Genoa, University of Liverpool, University of Munich, and University of Nebraska) participated. The teams developed 18 federates that simulated different tasks on the moon surface. The tasks included mining, an asteroid warning system, and transportation using rovers.

The authors' team developed a federate that simulates the erection of a facility on the moon surface. At the construction site, there were two cranes that were controlled from the Earth and were used to assemble the facility's modules based on a provided schedule and spatial data as seen in Figure 2 [37].

The DON federate, developed by NASA team [38], provided 3D visualization capabilities for the distributed simulation. Unlike previous project (mentioned in Sections 1.2 and 1.3), this project utilizes a COTS visualization engine which overcomes many of the drawbacks noticed in the previous project. However, the visualization paradigm was coupled to the HLA simulation, and it cannot be utilized outside this scope. Additionally, it does not provide querying capabilities which means each visualization scenario has to be prepared individually.

1.5. Lesson Learned and Research Motivation. The variety of applications shows how important visualization is in the construction domain. However, most of the visualization applications demonstrated earlier focus on a specific case study which limits the visualization applications' usage in different contexts and using them interchangeably is impossible as each one was customized for a specific scenario. For example, in the earthmoving case, to empty/fill or move a truck, the visualizer assumed a specific format that might not be used in other simulation models.

Additionally, the visualizer shows all data generated by these scenarios, which means that changing time resolution or level of detail will require rerunning the scenarios with a new configuration. Table 1 shows a summary of the characteristics and limitations of these visualizers.

Accordingly, this research tries to overcome these limitations by providing a visualization framework that can be used with different applications and case studies. It considers using semantic web technology to develop a general data store that merges data from different sources, and then SPARQL queries retrieve data related to a visualization 


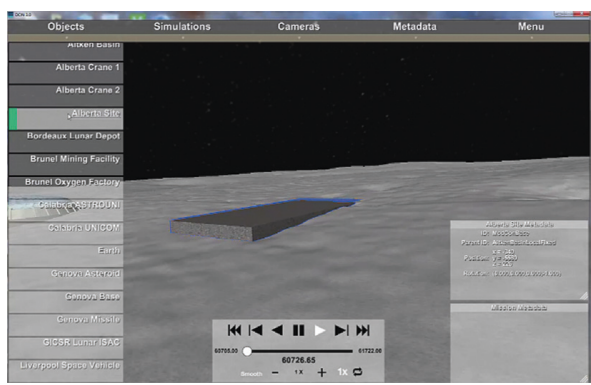

(a)

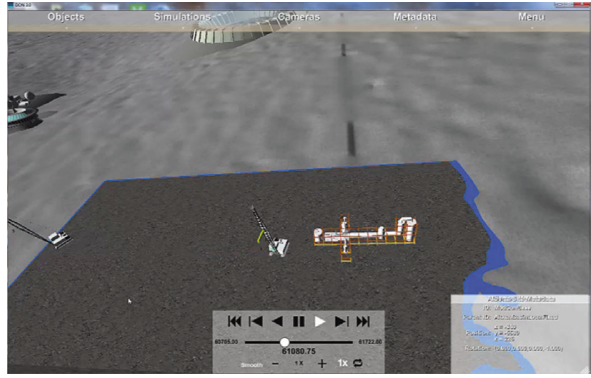

(c)

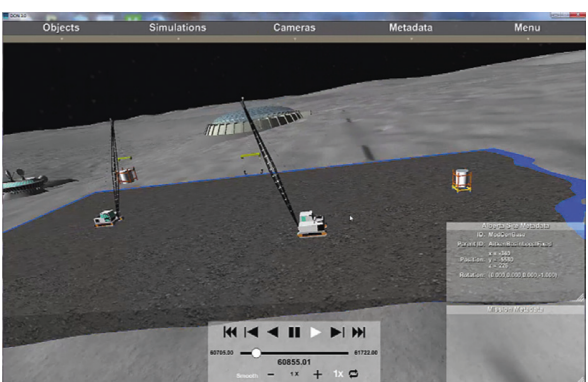

(b)

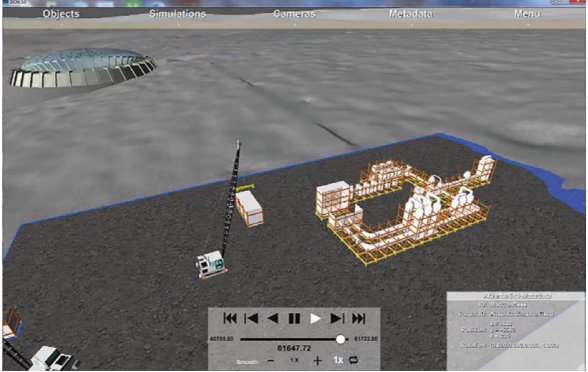

(d)

FIGURE 2: Erecting water treatment facility on the moon surface as rendered by DON.

TABLE 1: A summary of the characteristics of three developed visualization engines.

\begin{tabular}{|c|c|c|c|}
\hline & \multicolumn{3}{|c|}{ Visualizer } \\
\hline & Pipe manufacturing & Earthmoving & $\mathrm{DON}$ \\
\hline Coupled to a specific case & Yes & Yes & $\begin{array}{l}\text { Relatively, it works with distributed } \\
\text { simulations with a specific FOM }\end{array}$ \\
\hline $\begin{array}{l}\text { Underlaying visualization } \\
\text { engine }\end{array}$ & Open source engine & Built from scratch & Open source engine \\
\hline $\begin{array}{l}\text { Ability to display different } \\
\text { scenarios }\end{array}$ & $\begin{array}{l}\text { Requires rerunning } \\
\text { the simulation }\end{array}$ & $\begin{array}{l}\text { Requires rerunning } \\
\text { the simulation }\end{array}$ & Requires rerunning the simulation \\
\hline $\begin{array}{l}\text { Ability to visualize different } \\
\text { domains }\end{array}$ & No & No & Requires a new setup for this domain \\
\hline Merge new data source & $\begin{array}{l}\text { Has to be merged with } \\
\text { the simulation }\end{array}$ & $\begin{array}{l}\text { Has to be merged with } \\
\text { the simulation }\end{array}$ & Has to be merged with the simulation \\
\hline Querying & No & No & No \\
\hline
\end{tabular}

scenario and format it according to the corresponding visualizer input schema.

More specifically, the proposed approach tries to address the following questions:

(i) What are the main concepts to be included in the ontology? The key concepts and taxonomy in visualization-such as time, position, and orientation-must be carefully investigated.

(ii) Will the semantic web be able to merge different data sources and reformat them for visualization? The construction domain uses different applications, including schedule engines, relational databases, BIM, spreadsheets and, to lesser extent, simulation engines. Specialized connectors for some common applications that can retrieve the data and convert it to the proposed ontology schema will be developed. (iii) Can the stored (resource description framework) RDF data be formatted to the visualizer format? For now, the stream of data coming from different sources has been converted to the RDF format. Another connector is required to process this data and feed it to the visualizer to be shown.

(iv) Can the RDF format and its query engine enhance the visualization process by providing a way to query and display objects based on the required level of detail? Different audiences require different visualization levels of detail. For example, a project owner or an engineering firm might be interested in $4 \mathrm{D}$ or $5 \mathrm{D}$ visualization, while a contractor might be more interested in a more detailed visualization that shows operational activities such as crane movement and scaffold erection. These different scenarios require different time resolutions and objects. Our ontology should be able to 
store and show all these scenarios using a querying engine.

\section{Research Methodology}

The research methodology requires three main processes as shown in Figure 3: (1) combine the heterogeneous construction data into one data storage, (2) provide a query engine for the data storage, and (3) link the query results to a visualization engine. This procedure allows obtaining different visualization scenarios by changing the query statement. Additionally, the query is independent of the heterogeneous data sources as it runs on the merged data storage.

The following steps will be followed to pursue the proposed methodology:

(i) Evaluate previous work related to construction visualization.

(ii) List the capabilities required for the framework and how they can be achieved.

(iii) Propose the framework.

(iv) Implement the framework components.

(v) Test the framework with real-case scenarios.

2.1. Proposed Framework. Figure 4 shows the proposed framework for visualizing heterogeneous data coming from different data sources. The scope of work includes developing the ontology-backed by a triple store-preparing connectors that take raw data from different applications that are widely used in the construction domain and converting it to an $\mathrm{RDF}$ format and developing a hub that retrieves data from the triple store and converts it to the visualizer format as shown in Figure 4. Although one visualization application has been utilized, the same ontology can be used with different visualization applications with minimal effort to create a new connector.

The framework consists of two main parts: (1) existing components that are widely used in the construction domain and (2) developed components that stream data between different sources and the visualization engine. The following subsections discuss the developed components and their relationships with the existing components.

2.1.1. Ontology. Ontology is the key component in this framework as it formulates the relationship between data sources and the visualization. It should be generic enough to capture various data sources, but at the same time it should be structured to be able to export temporal and positional information.

Researchers suggested defining ontology requirements in the form of questions. These questions, which are known as "competency questions" determine the scope of the ontology [39]. Our competency questions are:

(i) Will the ontology be generic enough to receive data from different sources? (ii) Will it be able to convert the data to the visualization format?

(iii) Will it be able to filter by item type?

(iv) Will it be able to filter by levels?

(v) Will it be able to filter by different time resolutions?

(vi) Will it be able to filter by time intervals?

(vii) Will it be able to show temporary structures (such as a scaffold)?

First, to ensure interoperability, the ontology was built upon existing ontologies by importing ifcOWL [40] and W3C time ontology [41]. ifcOWL contains concepts and definitions related to BIM objects (e.g., walls and doors), while time ontology defines time concepts such as time positions, before, and after.

Next, the ontology defines a new class, named "Model Object," for any object that has to be shown in the visualizer. This class encompasses definitions for position coordinates and units, 3D orientation in a quaternion format, object scale, and file path for the 3D asset.

Any object from any data source has to inherit this class to be shown in the visualizer. For example, we asserted that "IFC4:IfcElement" (from ifcOWL) is a subclass of "Model Object." This means that if any instance in ifcOWL provides positional input and 3D asset's file path, the instance can be displayed in the visualizer.

The previous step will show a static 3D scene, and to add animation, different positional properties should be provided at key time frames. Hence, the ontology contains another class, "Object Time Stamp." This class captures the relationship between the model object and the time instance as defined in the W3C time ontology. In addition, this class contains information about object orientation and position at a specific time instant.

The ontology is backed by a triple store to handle the expected huge number of triples. Fuseki version 2.4.1 [42] has been used which can handle millions of triples and also provides a web-based interface and SPARQL endpoints that can be used to process the data.

2.1.2. Data Mapping (Data Sources to RDF). This section describes how data from different data sources can be exported to an RDF format based on the proposed ontology; this process is known as data integration [43]. It presents the integration of some of the common applications in the construction domain. However, it is worth to mention here that this list is not exhaustive, and additional data sources can be easily added.

(1) BIM Models. BIM models are widely used by engineering firms and in the construction domain to improve project management and collaboration [44]. In our context, BIM models provide rich information for visualization. This includes 3D assets and positional data. To export 3D assets from the 3D model, a customized plug-in that creates a separate OBJ file for each object in the model has been used. Afterwards, we used the visual programming tool 


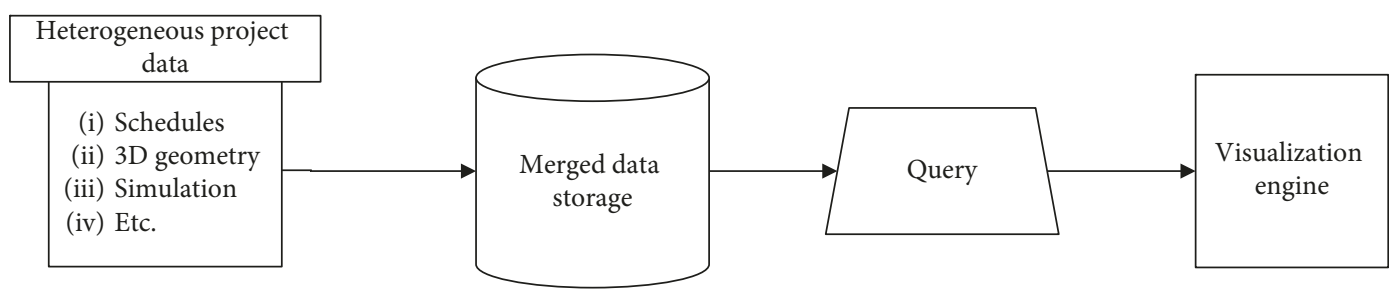

Figure 3: An overview of the proposed framework.

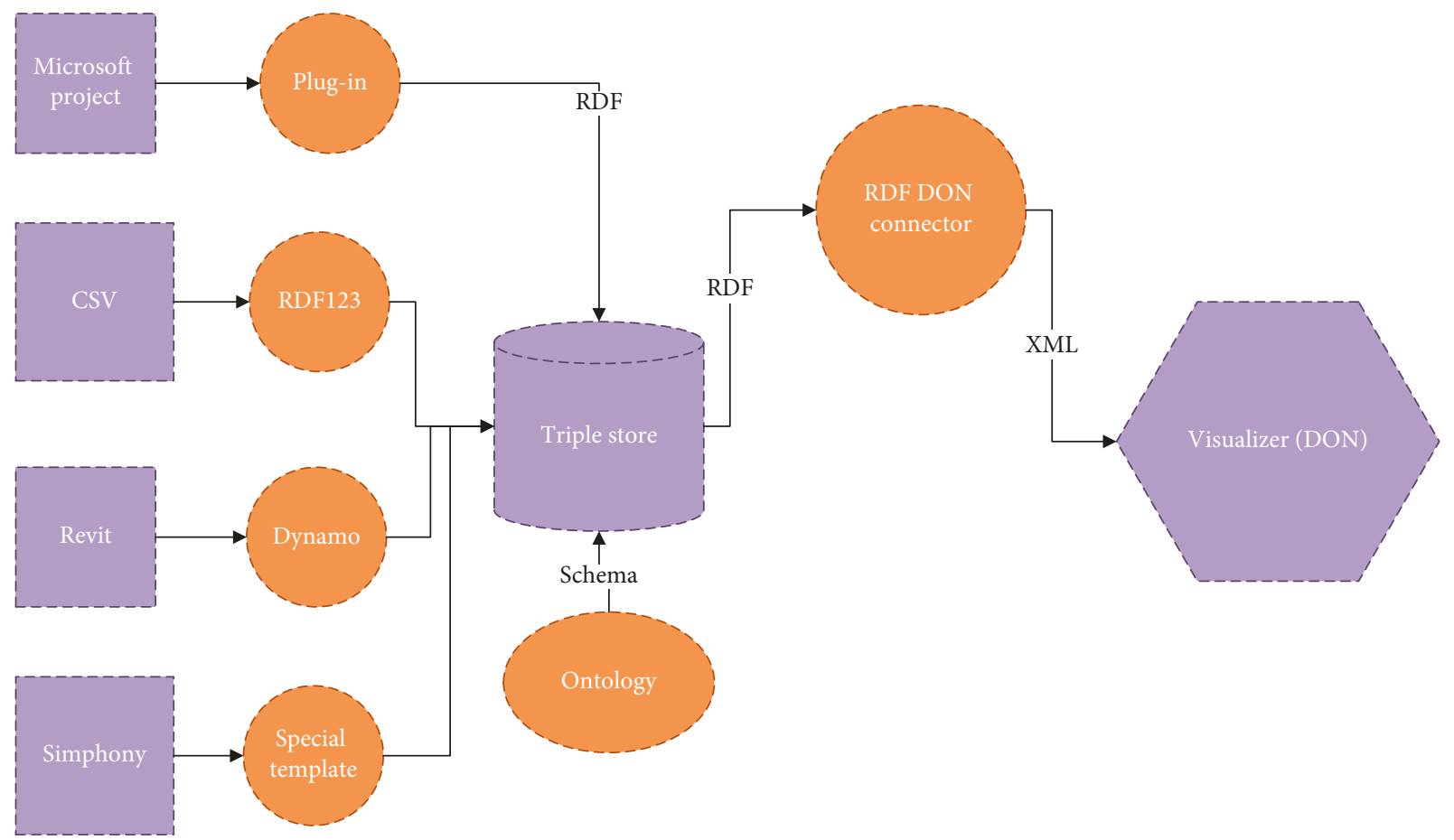

Proposed component

Third-party component

FIgURE 4: A schematic drawing of the proposed framework.

(Dynamo) [45-47] to export: (1) the location, (2) the orientation, and (3) OBJ file path for each object in the model. The data are exported in spreadsheet format which can be converted to RDF triples as will be shown later. The BIM models' connector has been tested with a steel structure frame, shown in Figure 5, which will be animated based on an associated construction schedule later.

(2) Schedules. Another key component of a construction project is the schedule. The schedule contains temporal information that can used to animate objects from other sources. A customized plug-in for Microsoft Project ${ }^{\circledR}$ (Figure 6) has been used to convert tasks finish/actual finish to the RDF format; this plug-in can write the RDF triples to a local file or a triple store through an HTTP connection. Clearly, an ID that links the task with items from other sources is required.
(3) Simulation Models. Simulation is a powerful tool to capture and model dynamic systems with a large number of variables that are hard to model using mathematical models. It provides an experimental frame for testing a real world system effectively and cheaply [48]. Visualization is arguably one of the most suitable ways to not only interpret results but validate and accredit the simulation model [49].

In our case, simulation models were used as another data source to give more details about a process modelled using RDF triples. For example, merging a BIM model with its associated schedule will create a stand-alone 4D visualization in which objects will appear in their final location when the associated tasks are complete. However, a simulation model allows for the addition of more details about object hauling from the storage area and crane lifting and swinging. 


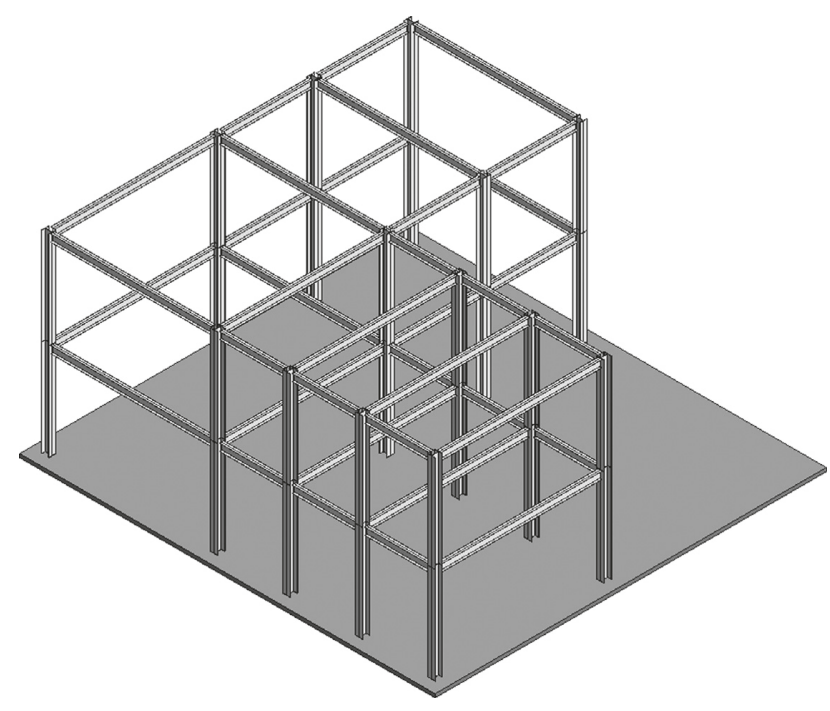

Figure 5: A sample BIM model for a steel structure frame.

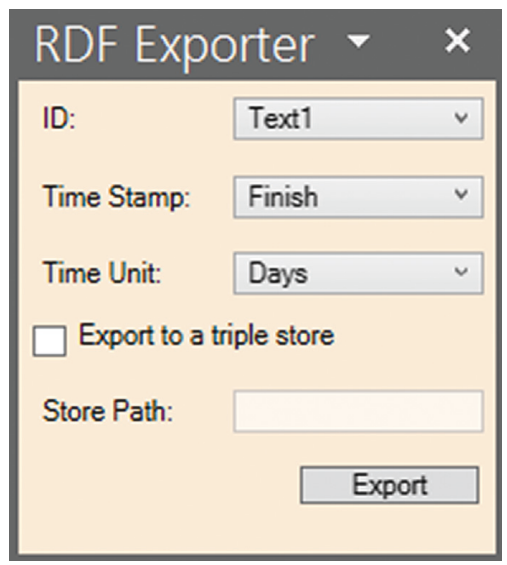

Figure 6: The interface of the schedule connectors.

A special simulation template in Simphony [50, 51] has been developed. It simulates the interactions between modules, trailers, and cranes. This template exports the results as RDF triples.

(4) Spreadsheets. Many data sources come in a spreadsheet format or at least can be converted to this format, such as data from BIM models as shown earlier. This section describes how the data in the format have been mapped to RDF triples.

RDF123 [52, 53] is an open-source tool that exports tabular data to an RDF format through a mapping graph. The mapping graph should be structured based on the spreadsheet structure and the ontology.

As an example of this conversion, a scaffold requests log for an oil and gas project in Alberta was obtained. The log is in a tabular data format with the following relevant columns: Request ID, Location, Required Elevation, Erection Date, and Dismantle Date.

This data are converted to RDF triples which were added to an existing $4 \mathrm{D}$ visualization to show scaffolds during the visualization process, as will be shown later.
2.1.3. RDF to Visualizer Connector. The previous section discussed our work regarding converting different data sources to the RDF format. Now, the generated RDF triples have to be converted to the visualizer application data format as shown in Figure 4. This requires a customized connector that converts from RDF to the visualizer format. This section describes the conversion from RDF triples to visualize them in DON.

DON accepts XML files as an input file [54]. In general, two XML files are required to visualize a process in DON. The first file is a "Mission File" which constructs the visualization scene by providing information about environment, cameras, lights, object hierarchy, and a reference for the second XML file [54]. The second XML file, known as the "Data File," contains two main sections: (1) initialization and (2) time section.

The initialization section contains metadata definitions and a list of objects that will be referenced in the time section. The time section captures the time steps in chronological order. Each time step might specify a new position or orientation for any object defined in the initialization section. The visualizer will interpolate the animation between each two consecutive time steps. The current XML schema of the input file is "MPC3," and it is documented in [54].

The authors developed a connector that takes RDF triples and converts them to XML files according to DON schema. The connector utilizes dotNetRDF to execute remote SPARQL queries and create the two XML files based on the query's results. This structure allows us to filter data based on customized queries as shown in the following section.

\section{Results}

After describing different data sources that have been used, this section illustrates how to merge data from different sources to get an animation for the whole process with different levels of detail. The following sections describe three scenarios. The first is a merger of a schedule with a BIM model to display 4D animation. The second adds scaffold erection and dismantling times and locations, while the third focuses on a finer time resolution and visualizes the handling of a module using a crane.

3.1. $4 D$. $4 \mathrm{D}$ animation is a high-level visualization that focuses on the big picture by showing the erection of a facility based on the actual or planned progress. Figure 7 shows a steel frame erection. Figure 8 shows module installations for an oil and gas project.

In both cases, the following steps have been executed: first, export the 3D assets from Revit in the first case and Blender in the second, and then, convert spatial information into an RDF format. Afterwards, map each item to the corresponding task in the schedule and convert the schedule to an RDF format. Finally, use the developed connector to create the XML files which are visualized in DON. 


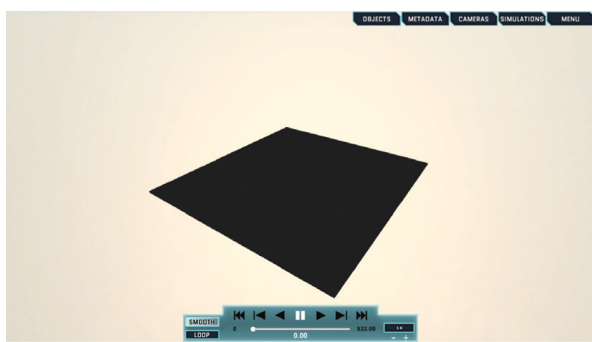

(a)

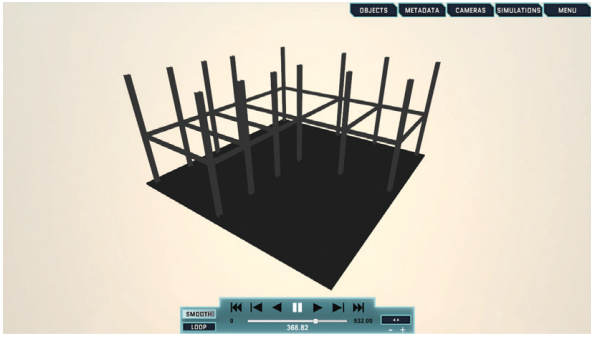

(c)

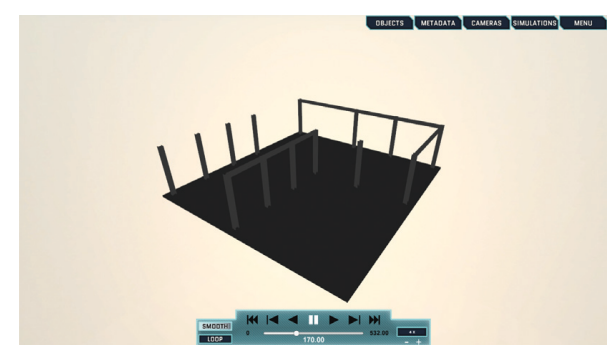

(b)

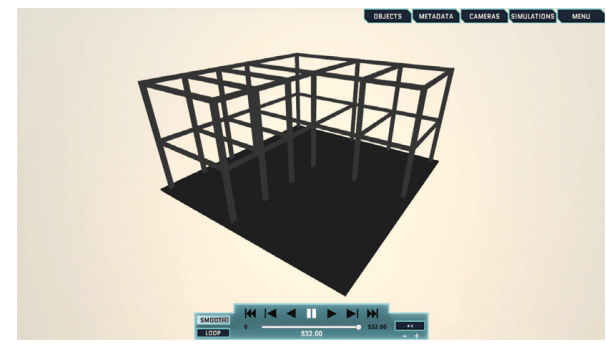

(d)

FIgURE 7: A sequence of screenshots that shows steel frame erection.

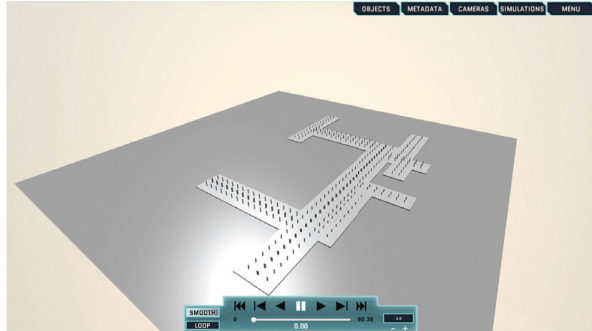

(a)

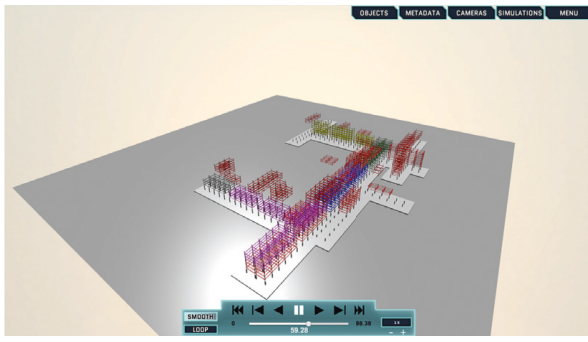

(c)

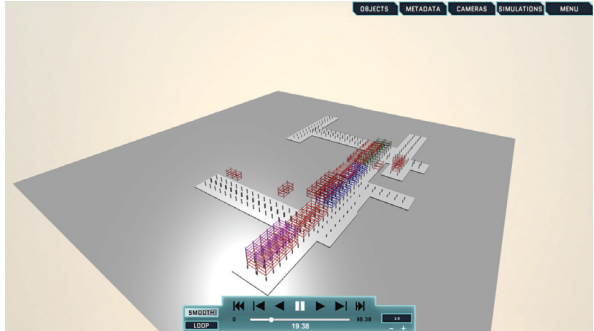

(b)

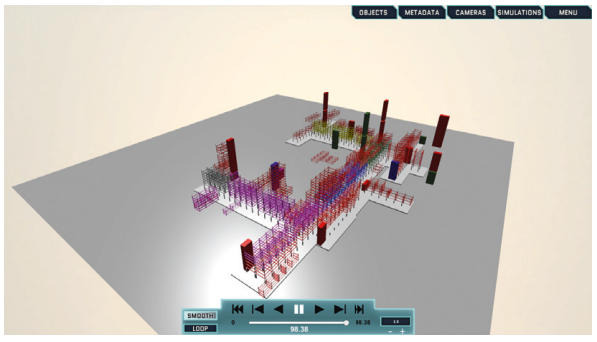

(d)

Figure 8: A sequence of screenshots that shows module installation in an oil and gas project.

3.2. 4D with Scaffold. This scenario demonstrates the advantages of the proposed framework as it automatically adds another data source (spreadsheet) to an existing visualization process without any required editing. The authors obtained the scaffold log for the same oil and gas project. The log is maintained by general foremen in the site. These are not the same personnel who maintained the $3 \mathrm{D}$ model. Nonetheless, merging these heterogeneous data sources went smoothly. Figure 9 shows that scaffolds were in the right place at the right time. Because 3D assets for the scaffold were not available, scaffold has been visualized as a transparent $3 \mathrm{D}$ box.
3.3. Crane Movement. The same framework can be used to show a more detailed visualization using simulation data as shown in this scenario. This scenario captures the module lifecycle starting from the storage area. A trailer moved the module to the pickup point. Afterwards, the crane lifted the module, swung it, moved to the drop point, and then dropped the module in the final location as shown in Figure 10.

\section{Limitations and Future Work}

The previous section demonstrates that the proposed framework is able to visualize different scenarios by merging 


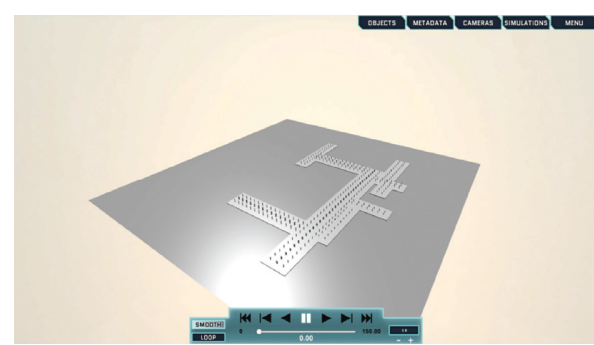

(a)

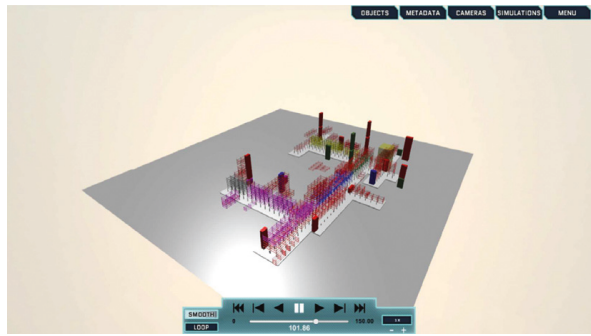

(c)

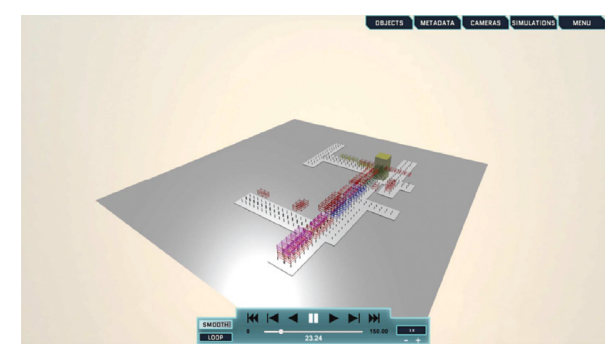

(b)

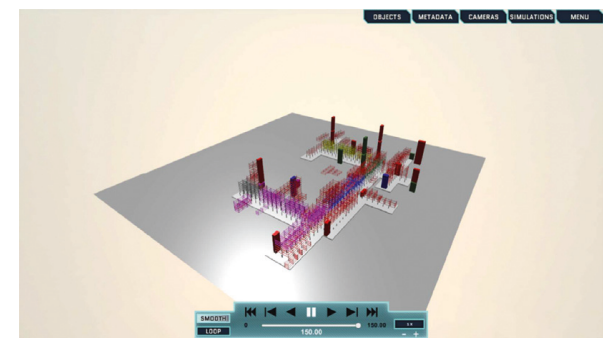

(d)

Figure 9: This is the same project shown in Figure 8, but we added the scaffold (the transparent objects in the second and third frame).
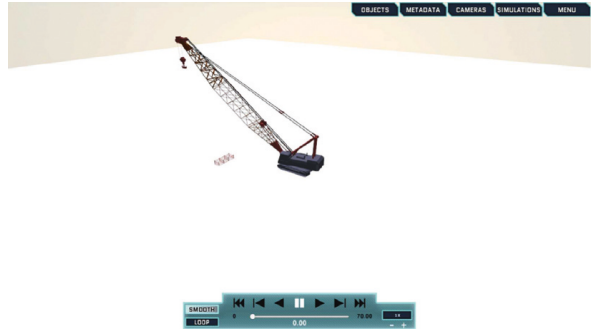

(a)
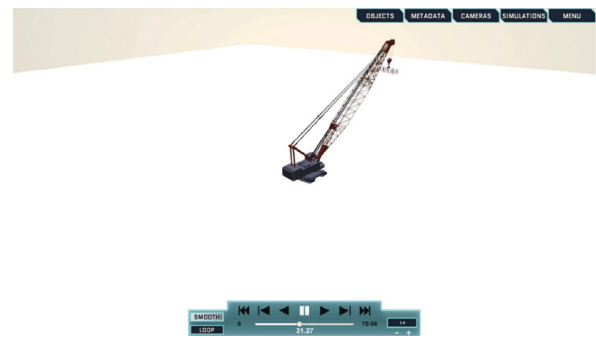

(c)
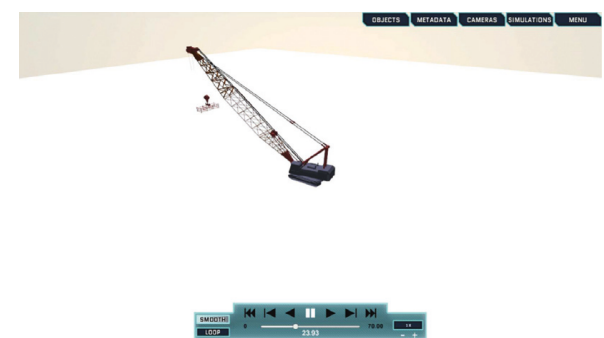

(b)

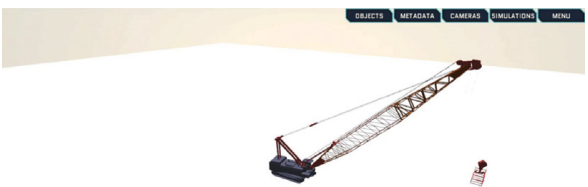

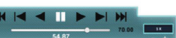

(d)

FIGURE 10: Handling of a module using a crane that shows lifting, swinging, hauling, and dropping.

heterogeneous data. Nevertheless, there are some limitations with this approach which includes the necessity of mapping different data to RDF format. This mapping carries two challenges. First, some data such as temporal data pose challenges in mapping to RDF [55-57]. Second, generated $\mathrm{RDF}$ triples are counted in millions which lengthen processing and querying time.

Additionally, using the same triple store for different level of details requires writing complex SPARQL queries to select triples related to a specific level of details. A future work will investigate adding more semantics to the generated triples to streamline filtering by the required level of details.

\section{Conclusion}

Visualization of construction activities is a complex process that requires significant effort to select the visualizer, prepare $3 \mathrm{D}$ assets, retrieve data, and transform it according to the visualizer specification. This ad hoc methodology led to the creation of many visualization models that are only suitable for one or two application cases. This paper presents a new framework for visualizing construction projects and activities. The framework focuses on the data rather than the visualization engine. By using an RDF data format as a data hub, data from different sources and formats can be merged 
into one triple store. This reduces the problem of visualization to the selection of a visualization engine and development of data bridges between the triple store and the visualizer and between the data sources and the triple store. The methodology is tested with different scenarios that demonstrated the ability to visualize different ranges of activities with different levels of detail.

\section{Data Availability}

The data are confidential as they are related to a construction company and cannot be shared.

\section{Disclosure}

This paper is part from the first author $\mathrm{PhD}$ thesis that is submitted to University of Alberta [58].

\section{Conflicts of Interest}

The authors declare that they have no conflicts of interest.

\section{Acknowledgments}

The authors would like to acknowledge the contribution of the Natural Science and Engineering Research Council of Canada (NSERC) for its funding of this research through the Collaborative Research and Development (CRD) Grant no. CRDPJ 416362-11.

\section{References}

[1] K. W. Chau, M. Anson, and J. P. Zhang, "Four-dimensional visualization of construction scheduling and site utilization," Journal of Construction Engineering and Management, vol. 130, no. 4, pp. 598-606, 2004.

[2] B. Koo and M. Fischer, "Feasibility study of 4D CAD in commercial construction," Journal of Construction Engineering and Management, vol. 126, no. 4, pp. 251-260, 2000.

[3] K. W. Chau, M. Anson, and J. P. Zhang, "Implementation of visualization as planning and scheduling tool in construction," Building and Environment, vol. 38, no. 5, pp. 713-719, 2003.

[4] V. R. Kamat, J. C. Martinez, M. Fischer, M. Golparvar-Fard, and S. Savarese, "Research in Visualization Techniques for Field Construction," Journal of Construction Engineering and Management, vol. 137, no. 10, pp. 853-862, 2011.

[5] M. Bilal, L. O. Oyedele, J. Qadir et al., "Big Data in the construction industry: A review of present status, opportunities, and future trends," Advanced Engineering Informatics, vol. 30 , no. 3, pp. 500-521, 2016.

[6] D. Heesom and L. Mahdjoubi, "Trends of 4D CAD applications for construction planning," Construction Management and Economics, vol. 22, no. 2, pp. 171-182, 2004.

[7] T. Berners-Lee, J. Hendler, and O. Lassila, "The semantic web: A new form of web content that is meaningful to computers will unleash a revolution of new possibilities," Scientific American, vol. 284, no. 5, pp. 35-43, 2001.

[8] The W3C SPARQL Working Group: n.d., "SPARQL 1.1 overview," January, 2017, https://www.w3.org/TR/sparql11overview/.
[9] V. R. Kamat and J. Martinez, "Visualizing Simulated Construction Operations in 3D," Journal of Computing in Civil Engineering, vol. 15, no. 4, pp. 329-337, 2001.

[10] S. M. AbouRizk, "Role of simulation in construction engineering and management," Journal of Construction Engineering and Management, vol. 136, no. 10, pp. 1140-1153, 2010.

[11] Y. Zhang, S. M. AbouRizk, H. Xie, and E. Moghani, “Design and implementation of loose-coupling visualization components in a distributed construction simulation environment with HLA," Journal of Computing in Civil Engineering, vol. 26, no. 2, pp. 248-258, 2012.

[12] S. Jain and C. R. McLean, "Integrated simulation and gaming architecture for incident management training," in Proceedings of the Winter Simulation Conference, pp. 904-913, Orlando, FL, USA, December 2005.

[13] S. Lee, D. Nikolic, J. I. Messner, and C. J. Anumba, "The development of the virtual construction simulator 3: an interactive simulation environment for construction management education," in Proceedings of International Workshop on Computing in Civil Engineering Computing in Civil Engineering, pp. 454-461, American Society of Civil Engineers, Miami, FL, USA, June 2011.

[14] M. Al-Hussein, M. Athar Niaz, H. Yu, and H. Kim, "Integrating $3 \mathrm{D}$ visualization and simulation for tower crane operations on construction sites," Automation in Construction, vol. 15, no. 5, pp. 554-562, 2006.

[15] S. C. Kang, H. L. Chi, and E. Miranda, "Three-dimensional simulation and visualization of crane assisted construction erection processes," Journal of Computing in Civil Engineering, vol. 23, no. 6, pp. 363-371, 2009.

[16] A. H. Behzadan and V. R. Kamat, "Visualization of construction graphics in outdoor augmented reality," in Proceedings of the 37th Conference on Winter Simulation, WSC '05, Winter Simulation Conference, pp. 1914-1920, Orlando, FL, December 2005.

[17] W. Ribarsky, J. Bolter, A. Op den Bosch, and R. van Teylingen, "Visualization and analysis using virtual reality," IEEE Computer Graphics and Applications, vol. 14, no. 1, pp. 10-12, 1994.

[18] T. Cheng and J. Teizer, "Real-time resource location data collection and visualization technology for construction safety and activity monitoring applications," Automation in Construction, Information Technologies in Safety Management, vol. 34, pp. 3-15, 2013.

[19] Y. Zhou, L. Y. Ding, and L. J. Chen, "Application of 4D visualization technology for safety management in metro construction," Automation in Construction, Information Technologies in Safety Management, vol. 34, pp. 25-36, 2013.

[20] K. A. Liapi, "4D visualization of highway construction projects," in Proceedings on Seventh International Conference on Information Visualization, IV 2003, pp. 639-644, London, UK, July 2003.

[21] M. L Pack, "Visualization in transportation: challenges and opportunities for everyone," IEEE Computer Graphics and Applications, vol. 30, no. 4, pp. 90-96, 2010.

[22] IEEE Standard for Modeling and Simulation (M\&S) High Level Architecture (HLA)-Federate Interface Specification, IEEE Std 1516.1-2010 (Revision of IEEE Std 1516.1-2000), Vol. 1, 2010.

[23] IEEE Standard for Modeling and Simulation (MßS) High Level Architecture (HLA)-Object Model Template (OMT) Specification, IEEE Std 1516.2-2010 (Revision of IEEE Std 1516.22000), 2010. 
[24] A. ElNimr and Y. Mohamed, "Loosely coupled visualization of industrial construction simulation using a gaming engine," in Proceedings of the 2011 Winter Simulation Conference (WSC), pp. 3577-3587, Phoenix, AZ, USA, December 2011.

[25] A. El Nimr and Y. Mohammed, "A simulation driven visualization framework for construction operations: development and application," in Proceedings of Construction Research Congress 2010, pp. 257-266, American Society of Civil Engineers, Banff, Canada, May 2010.

[26] S. Alkass, K. El-Moslmani, and M. AlHussein, "A computer model for selecting equipment for earthmoving operations using queuing theory," CIB Report, vol. 284, p. 1, 2003.

[27] M. Marzouk and O. Moselhi, "Object-oriented simulation model for earthmoving operations," Journal of Construction Engineering and Management, vol. 129, no. 2, pp. 173-181, 2003.

[28] Y. Mohamed and M. Ali, "A simplified online solution for simulation-based optimization of earthmoving operations," in Proceedings of 30th International Symposium on Automation and Robotics in Construction (ISARC 2013), Montreal, Canada, August 2013.

[29] M. Ali and Y. Mohamed, "Development of a model-based DSS for earth moving operations," in Proceedings of Al-Azhar Engineering Twelfth International Conference (AEIC 2012), Cairo, Egypt, 2012.

[30] G. Kannan, L. Schmitz, and C. Larsen, "An industry perspective on the role of equipment-based earthmoving simulation," in Proceedings of the 32nd Conference on Winter Simulation, WSC '00, pp. 1945-1952, Society for Computer Simulation International, Orlando, FL, USA, December 2000.

[31] S. Smith, J. Osborne, and M. Forde, "Analysis of earth-moving systems using discrete-event simulation," Journal of Construction Engineering and Management, vol. 121, no. 4, pp. 388-396, 1995.

[32] S. M. AbouRizk and K. Mather, "Simplifying simulation modeling through integration with 3D CAD," Journal of Construction Engineering and Management, vol. 126, no. 6, pp. $475-483,2000$.

[33] A. Montaser, M. Ibrahim, and O. Moselhi, "Adaptive forecasting in earthmoving operation using DES and site captured data," Procedia Engineering, vol. 85, pp. 377-384, 2014.

[34] F. Vahdatikhaki and A. Hammad, "Framework for near realtime simulation of earthmoving projects using location tracking technologies," Automation in Construction, vol. 42, pp. 50-67, 2014.

[35] M. Ali, M. Fagiar, Y. Mohamed, and S. M. AbouRizk, "Beyond classic models-design and development of a comprehensive earthmoving simulator," in Proceedings of 14th International Conference on Construction Applications of Virtual Reality in Construction and Conference on Islamic Architecture, Sharjah, UAE, November 2014.

[36] SEE Simulation Exploration Experience: "Simulation Exploration Experience (SEE)," n.d., March, 2017, http://www. exploresim.com/.

[37] M. Fagiar, M. Ali, and Y. Mohamed, "Capitalizing on visualizing complex systems using distributed simulation approach," in Proceedings of 15th International Conference on Construction Applications of Virtual Reality in Construction, Banff, Canada, October 2015.

[38] M. Conroy, R. Mazzone, W. Little et al., "Distributed observer network," NASA Tech Brief, vol. 1, 2010.

[39] M. Grüninger and M. S. Fox, "Methodology for the design and evaluation of ontologies," in Proceedings of Workshop on Basic
Ontological Issues in Knowledge Sharing, Montreal, Canada, August 1995.

[40] buildingSMART, “buildingSMART,” November 2016, http:// buildingsmart.org/.

[41] S. Cox and C. Little, "Time ontology in OWL," November 2016, https://www.w3.org/TR/owl-time/.

[42] Apache Jena-Fuseki: serving RDF data over HTTP," n.d., January 2017, https://jena.apache.org/documentation/ serving_data/.

[43] H. Kondylakis and D. Plexousakis, "Ontology evolution without tears," Web Semantics: Science, Services and Agents on the World Wide Web, vol. 19, pp. 42-58, 2013.

[44] C. Eastman, P. Teicholz, R. Sacks, and K. Liston, BIM Handbook: A Guide to Building Information Modeling for Owners, Managers, Designers, Engineers and Contractors, Wiley, Hoboken, NJ, USA, 2011.

[45] M. R. Asl, M. Bergin, A. Menter, and W. Yan, "BIM-based parametric building energy performance multi-objective optimization," Education and Research in Computer Aided Architectural Design in Europe, vol. 32, pp. 1-10, 2014.

[46] “Dynamo BIM," n.d., March 2017, http://dynamobim.org/.

[47] P. Janssen and K. W. Chen, "Visual dataflow modelling," in Proceedings of CAAD Futures, pp. 801-816, Liège, Belgium, 2011.

[48] B. P. Zeigler, H. Praehofer, and T. G. Kim, Theory of Modeling and Simulation: Integrating Discrete Event and Continuous Complex Dynamic Systems, Academic Press, Cambridge, MA, USA, 2000.

[49] V. R. Kamat and J. C. Martinez, "Validating complex construction simulation models using 3D visualization," Systems Analysis Modelling Simulation, vol. 43, no. 4, pp. 455-467, 2003.

[50] S. AbouRizk and Y. Mohamed, "Simphony-an integrated environment for construction simulation," in Proceedings of 2000 Winter Simulation Conference (Cat. No.00CH37165), vol. 2, pp. 1907-1914, Orlando, FL, USA, December 2000.

[51] D. Hajjar and S. AbouRizk, "Simphony: an environment for building special purpose construction simulation tools," in Proceedings of the 31st Conference on Winter Simulation: Simulation-a Bridge to the Future-Volume 2, WSC '99, pp. 998-1006, ACM, Phoenix, AZ, USA, 1999.

[52] L. Han, T. Finin, C. Parr, J. Sachs, and A. Joshi, "RDF123: a mechanism to transform spreadsheets to RDF," in Proceedings of the Twenty-First National Conference on Artificial Intelligence, AAAI Press, Menlo ParkBoston, MA, USA, July 2006.

[53] L. Han, T. Finin, C. Parr, J. Sachs, and A. Joshi, RDF123: From Spreadsheets to RDF, The Semantic Web-ISWC 2008, Springer, Berlin, Heidelberg, 2008.

[54] R. A. Mazzone and M. P. Conroy, Data Presentation and Visualization (DPV) Interface Control Document, 2015.

[55] I. Davis, "Representing time in RDF part 1," Internet Alchemy, November 2016, http://blog.iandavis.com/2009/08/ representing-time-in-rdf-part-1/.

[56] C. Gutierrez, C. A. Hurtado, and A. Vaisman, "Introducing Time into RDF," IEEE Transactions on Knowledge and Data Engineering, vol. 19, no. 2, pp. 207-218, 2007.

[57] C. Gutierrez, C. Hurtado, and A. Vaisman, Temporal RDF, SpringerLink, Springer, Heidelberg, Germany, 2005.

[58] M. Ali, Extracting and integrating industrial construction steel trade data in ill-formed BIM models, Ph.D. Thesis, University of Alberta, Edmonton, Canada, 2017. 


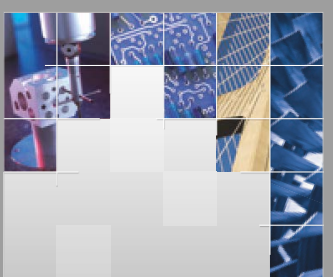

\section{Enfincering}
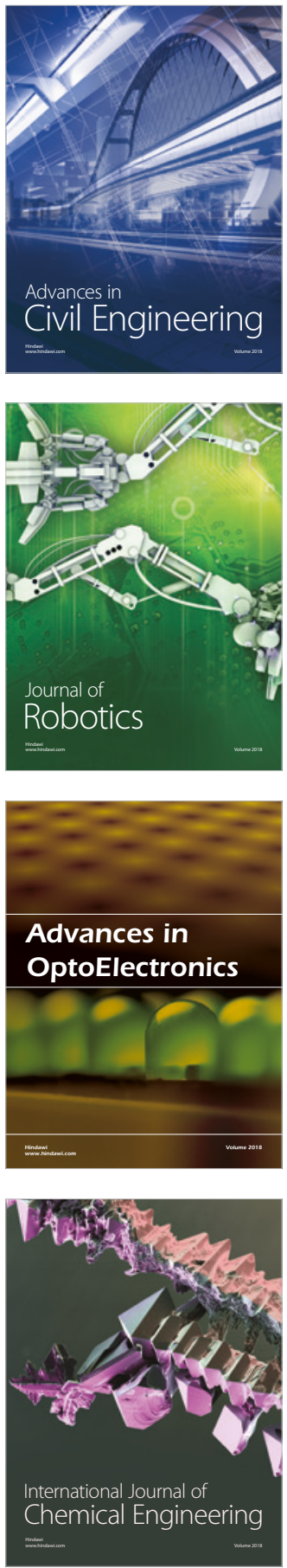

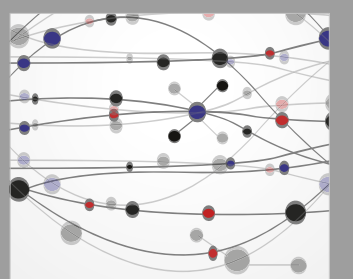

\section{Rotating \\ Machinery}

The Scientific World Journal

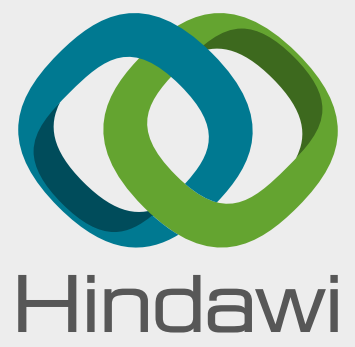

Submit your manuscripts at

www.hindawi.com
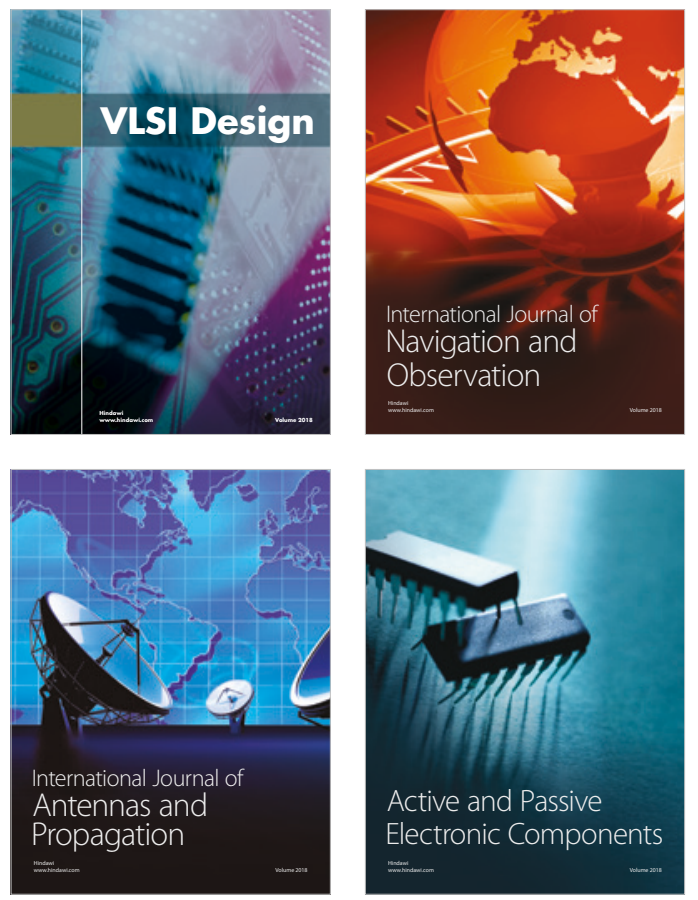
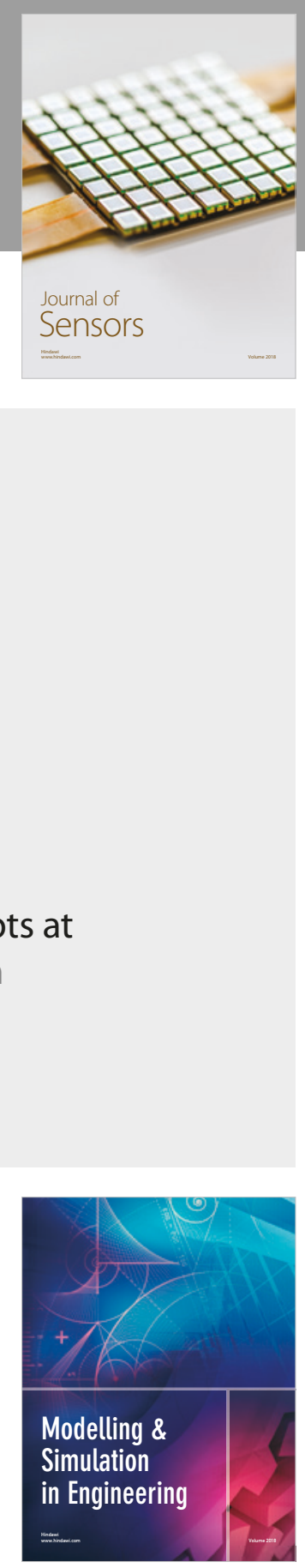

\section{Advances \\ Multimedia}
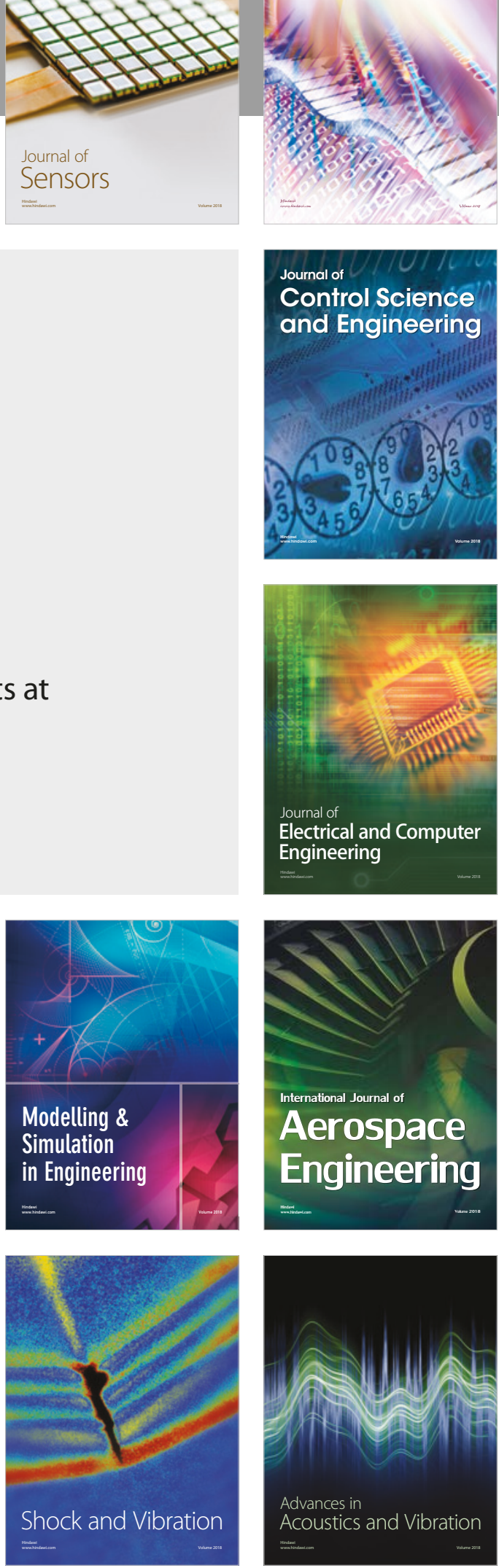